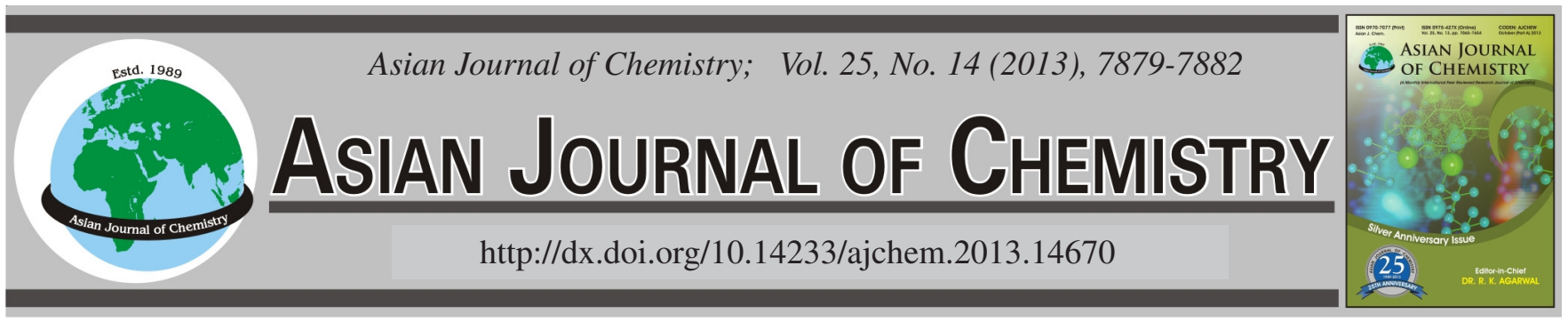

\title{
Synthesis of Pyrazoleacrylic Acids and Their Derivatives
}

Faryal Chaudhry ${ }^{1}$, Nadia Asif ${ }^{1}$, Muhammad Naeem Khan $^{3, *}$, Jorge Ribeiro $^{2, \dagger}$, Abdul Qayyum Ather ${ }^{3}$, Sajila Hina $^{4}$, Munawar Ali Munawar ${ }^{1}$, Muhammad Nasrullah ${ }^{5}$, Qura Tul Ain ${ }^{1}$, Farah Suhail $^{1}$ and Misbahul Ain Khan ${ }^{1,2,5}$

${ }^{1}$ Institute of Chemistry, University of the Punjab, Quaid-e-Azam Campus, Lahore, Pakistan

${ }^{2}$ Secao de Quimica, Instituto Militar de Engenharia, Praia Vermelha, Rio de Janeiro, Brasil

${ }^{3}$ Applied Chemistry Research Centre, PCSIR Laboratories Complex, Lahore, Pakistan

${ }^{4}$ Food and Biotechnology Research Centre, PCSIR Laboratories Complex, Lahore, Pakistan

${ }^{5}$ Department of Chemistry, The Islamia University of Bahawalpur, Bahawalpur, Pakistan

*Corresponding author: E-mail: changwani_1@yahoo.com

$\dagger$ Deceased

(Received: 11 October 2012;

Accepted: 22 July 2013)

AJC-13832

Various pyrazole acrylic acid derivatives were obtained by the Knoevenagel condensation of pyrazole-4-carbaldehydes with 'active methylene' compounds. In the condensation reaction with malonic acid and cyanoacetic acid, decarboxylation of the resulting acids was not observed. The products were characterized through spectroscopic techniques and elemental analysis.

Key Words: Pyrazoles, Knoevenagel condensation, Pyrazole-4-carbaldehydes.

\section{INTRODUCTION}

Knoevenagel condensation ${ }^{1}$ is a very effective way of extending carbon chains and often exploited for the purpose. Pyrazole chemistry has afforded some interesting biological materials; one of these is COX-2 inhibitor i.e., celecoxib ${ }^{2}$ (a), already in clinical use, other is a well known agrochemical i.e., furamtpyr ${ }^{3}$ (b), used as a fungicide. In another azole researches such as with pyrrole some interesting pyrrole-3acetic acid derivatives are being explored as NSAIDs ${ }^{4}$. Similar acid derivatives could easily be obtained from pyrazole-4carbaldehydes.

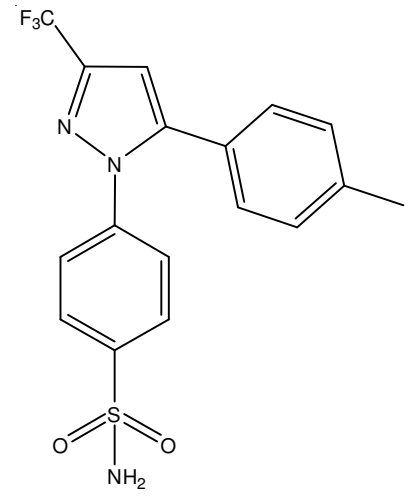

(a) Celecoxib

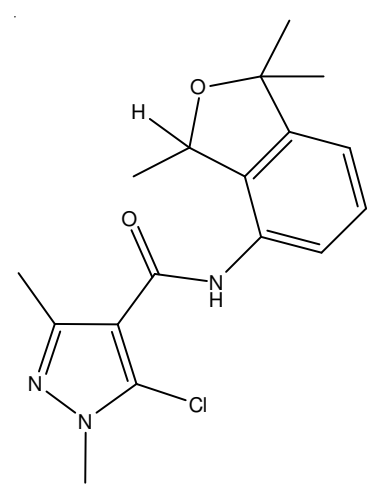

(b) Furamptyr
Fig. 1. Important pyrazole containing biological compounds
An appropriate pyrazolecarbaldehyde through Knoevenagel condensation could provide such a scaffold for posterior manipulation. Pyrazole-4-carbaldehydes are readily obtainable from various reactions such as: by the Rosenmund reduction of pyrazole-4-carbonyl chloride ${ }^{5 a, b}$, by the Sommelet reaction of the corresponding 4-chloromethylpyrazole ${ }^{5 c}$, by the Vilsmeier-Haack reaction of pyrazoles ${ }^{6-9}$ or by the reaction of appropriate semicarbazones or hydrazones with Vilsmeier reagent ${ }^{10,11}$. Recently, a review on 1,3-diarylpyrazol-4-carbaldehyde has appeared in literature ${ }^{12}$. Some of these aldehydes have previously been condensed with various substrates such as acetophenones ${ }^{7,13}, 4$-acetylpyrazole ${ }^{14}$, pyruvic acid ${ }^{15}$, hippuric $\operatorname{acid}^{16}$, malonic acid ${ }^{7,17}$, indole-3-acetonitrile ${ }^{18}$ and benzopyrillium salts ${ }^{19}$.

These considerations prompt us to continue our work on pyrazole chemistry and we have already reported some of our work on pyrazole derivatives ${ }^{20}$. Now we would like to present our results of Knoevenagel condensations of some pyrazole4-carbaldehydes.

\section{EXPERIMENTAL}

All the chemicals were purchased from Aldrich and used without further purification. The ${ }^{1} \mathrm{H}$ NMR spectra were taken on a Hitachi Perkin-Elmer spectrometer model R-20B operating at $60 \mathrm{MHz}$ (tetramethylsilane as internal reference). The IR absorption spectra were recorded as potassium bromide discs on a Perkin-Elmer model 180 spectrophotometer. Elemental 
analysis was carried out on a Perkin-Elmer model 240. Melting points were obtained on a Fisher-Johns apparatus and are uncorrected.

Pyrazole-4-carbaldehydes: The following starting materials were prepared according to the literature methods: 1-Phenylpyrazole-4-carbaldehyde ${ }^{21}$ (1): m.p. 76-78 ${ }^{\circ} \mathrm{C}$, yield $85 \%$. 1-p-Methoxyphenylpyrazole-4-carbaldehyde ${ }^{22}$ (2): m.p. 83-84 ${ }^{\circ} \mathrm{C}$, yield $89 \%$. 3-Phenylpyrazole-4-carbaldehyde ${ }^{11}$ (3): m.p. $128-129{ }^{\circ} \mathrm{C}$, yield $87 \%$. 1-p-Nitrophenylpyrazole-4carbaldehyde $^{23}$ (4): m.p. $167-168^{\circ} \mathrm{C}$, yield $78 \%$.

\section{Knoevenagal condensations}

General procedure: A mixture of $0.01 \mathrm{~mol}$ of the aldehyde, $0.01 \mathrm{~mol}$ of the "active methylene" compound, three drops of piperidine and five drops of pyridine in $10 \mathrm{~mL}$ of ethanol was heated under reflux for the required time as monitored by TLC. The reaction mixture was cooled and inverted over crushed ice, acidified with hydrochloric acid ( $\mathrm{pH}$ 6), filtered, washed with cold water and dried. The dried precipitates were crystallized from an appropriate solvent. The following products (Scheme-I). were synthesized: 2-((1-Phenyl-1H-pyrazol-4yl)methylene)malonic acid (10): m.p. $206-207^{\circ} \mathrm{C}$, yield 1.93 g; $75 \%$. 2-((1-(4-Methoxyphenyl)-1H-pyrazol-4-yl)methylene)malonic acid (11): m.p. $165-166^{\circ} \mathrm{C}$, yield $2.12 \mathrm{~g}$; $74 \%$. 2-((3-Phenyl-1H-pyrazol-4-yl)methylene)malonic acid (12): m.p. $169^{\circ} \mathrm{C}$, yield $1.93 \mathrm{~g} ; 75 \%$. 2-((1-(4-Nitrophenyl)$1 H$-pyrazol-4-yl)methylene)malonic acid (13): m.p. 204$206^{\circ} \mathrm{C}$, yield $2.02 \mathrm{~g} ; 67 \%$. Diethyl 2-((1-phenyl-1H-pyrazol4-yl)methylene)malonate $(\mathbf{1 4})^{24}$ : m.p. $57-58{ }^{\circ} \mathrm{C}$, yield $2.34 \mathrm{~g}$; $75 \%$. Diethyl 2-((1-(4-methoxyphenyl)-1H-pyrazol-4yl)methylene)malonate (15): m.p. $74-75^{\circ} \mathrm{C}$, yield $2.43 \mathrm{~g} ; 71 \%$. Diethyl 2-((1-(4-nitrophenyl)-1H-pyrazol-4-yl)methylene)malonate (16): m.p. $128-129^{\circ} \mathrm{C}$, yield; $2.47 \mathrm{~g}$; $69 \%$. (Z)-2Phenyl-3-(1-phenyl-1H-pyrazol-4-yl)acrylonitrile (17): m.p. 121-122 ${ }^{\circ} \mathrm{C}$, yield $1.63 \mathrm{~g} ; 57 \%$. (Z)-3-(1-(4-Methoxyphenyl)$1 H$-pyrazol-4-yl)-2-phenylacrylonitrile (18): m.p. $169^{\circ} \mathrm{C}$, yield 2.14 g; 68 \%. (Z)-3-(1-(4-Nitrophenyl)-1H-pyrazol-4-yl)-

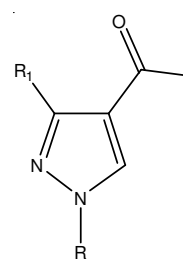

(1 - 4)

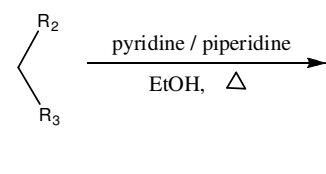

(5 - 9)

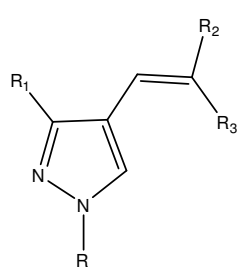

(10 - 27)
$\mathrm{R}=\mathrm{H}, \mathrm{Ph}, \mathrm{p}-\mathrm{MeOC}_{6} \mathrm{H}_{4}, \mathrm{p}-\mathrm{NO}_{2} \mathrm{C}_{6} \mathrm{H}_{4} ; \mathrm{R}_{1}=\mathrm{H}, \mathrm{Ph} ; \mathrm{R}_{2}=\mathrm{Ph}, \mathrm{COOH}, \mathrm{COOEt}, \mathrm{CN}$ $\mathrm{R}_{3}=\mathrm{COOH}, \mathrm{COOEt}, \mathrm{CN}$

Scheme-I

2-phenylacrylonitrile (19): m.p. 276-278 ${ }^{\circ} \mathrm{C}$, yield $2.81 \mathrm{~g}$; $85 \%$. (E)-Ethyl 2-cyano-3-(1-phenyl-1H-pyrazol-4yl)acrylate (20): m.p. $132-133{ }^{\circ} \mathrm{C}$, yield; 2.20 g; $83 \%$. (E)Ethyl 2-cyano-3-(1-(4-methoxyphenyl)-1H-pyrazol-4yl)acrylate (21): m.p. $151-152{ }^{\circ} \mathrm{C}$, yield $0.88 \mathrm{~g} ; 30 \%$. (E)Ethyl 2-cyano-3-(3-phenyl-1H-pyrazol-4-yl)acrylate (22): m.p. 167-168 ${ }^{\circ} \mathrm{C}$, yield $0.72 \mathrm{~g} ; 27 \%$. (E)-Ethyl 2-cyano-3-(1(4-nitrophenyl)-1H-pyrazol-4-yl)acrylate (23): m.p. 187-189 ${ }^{\circ} \mathrm{C}$, yield $0.87 \mathrm{~g} ; 28 \%$. (E)-2-Cyano-3-(1-phenyl-1H-pyrazol4-yl)acrylic acid (24): m.p. $212-214{ }^{\circ} \mathrm{C}$, yield $1.76 \mathrm{~g} ; 74 \%$. (E)-2-Cyano-3-(1-(4-methoxyphenyl)-1H-pyrazol-4yl)acrylic acid (25): m.p. 236-238 ${ }^{\circ} \mathrm{C}$, yield $2.19 \mathrm{~g} ; 82 \%$. (E)2-Cyano-3-(3-phenyl-1H-pyrazol-4-yl)acrylic acid (26): m.p. $249^{\circ} \mathrm{C}$, yield; $1.99 \mathrm{~g} ; 84 \%$. (E)-2-Cyano-3-(1-(4-nitrophenyl)$1 H$-pyrazol-4-yl)acrylic acid (27): m.p. $320-322^{\circ} \mathrm{C}$, yield; 2.26 g; $80 \%$. The results are presented in Tables 1 and 2 .

\section{RESULTS AND DISCUSSION}

We had already reported the synthesis of various pyrazole4-carbaldehydes and their derivatives ${ }^{25}$. The results of the present Knoevenagel condensations between the pyrazole-4carboxaldehydes and the "active methylene" compounds are presented in Scheme-I. A mixture of pyridine and piperidine was used in these condensations ${ }^{1}$. The reactions were carried out in refluxing ethanol. The results are collected in Table-1. Table- 2 contains the spectral data for the compounds obtained

\begin{tabular}{|c|c|c|c|c|c|c|c|}
\hline \multirow{3}{*}{ Compd. No. } & \multicolumn{6}{|c|}{$\begin{array}{c}\text { TABLE-1 } \\
\text { PHYSICAL DATA OF PRODUCTS } \\
\end{array}$} & \\
\hline & \multirow{2}{*}{ m.p. $\left({ }^{\circ} \mathrm{C}\right)$} & \multirow{2}{*}{$\begin{array}{l}\text { Solvents for } \\
\text { crystallization }\end{array}$} & \multirow{2}{*}{ Yield $(\%)$} & \multirow{2}{*}{ m.f. } & \multicolumn{3}{|c|}{ Elemental analysis (\%): calcd. (found) } \\
\hline & & & & & $\mathrm{C}$ & $\mathrm{H}$ & $\mathrm{N}$ \\
\hline 10 & $206-7$ & Chloroform & 75 & $\mathrm{C}_{13} \mathrm{H}_{10} \mathrm{~N}_{2} \mathrm{O}_{4}$ & $60.46(60.68)$ & $3.90(4.06)$ & $10.85(11.05)$ \\
\hline 11 & $165-6$ & Chloroform & 74 & $\mathrm{C}_{14} \mathrm{H}_{12} \mathrm{~N}_{2} \mathrm{O}_{5}$ & $58.33(57.97)$ & $4.20(4.36)$ & $9.72(9.34)$ \\
\hline 12 & 169 & Acetic acid & 75 & $\mathrm{C}_{13} \mathrm{H}_{10} \mathrm{~N}_{2} \mathrm{O}_{4}$ & $60.46(60.11)$ & $3.90(3.73)$ & $10.85(10.47)$ \\
\hline 13 & 204-6 & $\mathrm{DMF}^{\mathrm{b}}$ & 67 & $\mathrm{C}_{13} \mathrm{H}_{9} \mathrm{~N}_{3} \mathrm{O}_{6}$ & $51.49(51.56)$ & $2.99(2.94)$ & $13.86(14.22)$ \\
\hline 14 & $(57)^{24}$ & Ethanol & 75 & $\mathrm{C}_{17} \mathrm{H}_{18} \mathrm{~N}_{2} \mathrm{O}_{4}$ & $64.96(65.05)$ & $5.77(5.81)$ & $8.91(8.72)$ \\
\hline 15 & $74-75$ & Ethanol & 71 & $\mathrm{C}_{18} \mathrm{H}_{20} \mathrm{~N}_{2} \mathrm{O}_{5}$ & $62.73(62.68)$ & $5.85(5.68)$ & 8.14 (7.98) \\
\hline 16 & $128-9$ & Acetic acid & 69 & $\mathrm{C}_{17} \mathrm{H}_{17} \mathrm{~N}_{3} \mathrm{O}_{6}$ & $56.82(56.54)$ & 4.77 (4.59) & $11.69(11.62)$ \\
\hline $17^{\mathrm{c}}$ & $121-2$ & Ethanol & 57 & $\mathrm{C}_{18} \mathrm{H}_{13} \mathrm{~N}_{3}$ & $79.68(79.44)$ & $4.83(4.73)$ & 15.49 (15.23) \\
\hline $18^{\mathrm{c}}$ & 169 & Chloroform & 68 & $\mathrm{C}_{19} \mathrm{H}_{15} \mathrm{~N}_{3} \mathrm{O}$ & $75.73(75.58)$ & $5.02(5.07)$ & $13.94(13.88)$ \\
\hline $19^{c}$ & $276-8$ & Acetic acid & 85 & $\mathrm{C}_{18} \mathrm{H}_{12} \mathrm{~N}_{4} \mathrm{O}_{2}$ & $68.35(68.80)$ & $3.82(3.84)$ & $17.71(17.92)$ \\
\hline 20 & $132-3$ & Aq. Ethanol & 83 & $\mathrm{C}_{15} \mathrm{H}_{13} \mathrm{~N}_{3} \mathrm{O}_{2}$ & $67.40(67.18)$ & $4.90(4.80)$ & $15.72(15.87)$ \\
\hline 21 & $151-2$ & Ethanol & 30 & $\mathrm{C}_{16} \mathrm{H}_{15} \mathrm{~N}_{3} \mathrm{O}_{3}$ & $64.64(64.45)$ & $5.08(5.03)$ & $14.13(14.03)$ \\
\hline 22 & $167-8$ & Ethanol & 27 & $\mathrm{C}_{15} \mathrm{H}_{13} \mathrm{~N}_{3} \mathrm{O}_{2}$ & $67.40(67.52)$ & $4.90(4.84)$ & $15.72(16.00)$ \\
\hline 23 & $187-9$ & Ethanol & 28 & $\mathrm{C}_{15} \mathrm{H}_{12} \mathrm{~N}_{4} \mathrm{O}_{4}$ & $57.69(57.53)$ & 3.87 (3.69) & $17.94(17.83)$ \\
\hline $24^{\mathrm{d}}$ & $212-4$ & Chloroform & 74 & $\mathrm{C}_{13} \mathrm{H}_{9} \mathrm{~N}_{3} \mathrm{O}_{2}$ & $65.26(65.50)$ & $3.79(3.86)$ & $17.56(17.54)$ \\
\hline $25^{\mathrm{d}}$ & $236-8$ & Acetic acid & 82 & $\mathrm{C}_{14} \mathrm{H}_{11} \mathrm{~N}_{3} \mathrm{O}_{3}$ & $62.45(62.30)$ & $4.12(4.15)$ & $15.61(15.43)$ \\
\hline $26^{\mathrm{d}}$ & 249 & Ethanol & 84 & $\mathrm{C}_{13} \mathrm{H}_{9} \mathrm{~N}_{3} \mathrm{O}_{2}$ & $65.27(65.27)$ & $3.79(3.96)$ & $17.57(17.34)$ \\
\hline $27^{\mathrm{d}}$ & $320-2$ & $\mathrm{DMF}^{\mathrm{b}}$ & 80 & $\mathrm{C}_{13} \mathrm{H}_{8} \mathrm{~N}_{4} \mathrm{O}_{4}$ & $54.93(54.76)$ & $2.84(2.93)$ & $19.71(19.73)$ \\
\hline
\end{tabular}

${ }^{a}$ see Scheme-I; ${ }^{b}$ dimethyl formamide; ${ }^{c}$ reaction time $6 \mathrm{~h}$; ${ }^{\mathrm{d}}$ reaction time $10 \mathrm{~h}$. 


\begin{tabular}{|c|c|c|}
\hline \multicolumn{3}{|c|}{$\begin{array}{c}\text { TABLE-2 } \\
\text { SPECTROSCOPIC DATA OF PRODUCTS }^{\mathrm{a}}\end{array}$} \\
\hline Compd. No. & $\operatorname{IR}\left(v_{\max }, \mathrm{cm}^{-1}\right)^{\mathrm{b}}$ & ${ }^{1} \mathrm{H}$ NMR $\delta$ in $\operatorname{ppm}(J$ in $\mathrm{Hz})$ (solvent) \\
\hline 10 & $\begin{array}{l}3000-2500 \text { (br., } \mathrm{OH}), 1700 \text { and } 1675(\mathrm{C}=\mathrm{O}), 1645,1580, \\
1520,1490,1480,1450,1265,1200,1180,755,730 .\end{array}$ & $\begin{array}{l}7.60(\mathrm{~m}, 5 \mathrm{H}, \mathrm{Ar}), 8.50(\mathrm{~s}, 1 \mathrm{H}, \mathrm{CH}=\mathrm{C}), 8.92(\mathrm{~s}, 1 \mathrm{H}, \mathrm{H}-3), 9.07(\mathrm{~s}, 1 \mathrm{H}, \\
\mathrm{H}-5),\left(\mathrm{CF}_{3} \mathrm{COOH}\right)\end{array}$ \\
\hline 11 & $\begin{array}{l}3200-2500(\mathrm{br} ., \mathrm{OH}), 1710 \text { and } 1675(\mathrm{C}=\mathrm{O}), 1640,1580, \\
1520,1380,1260,1180,1015,825,725 .\end{array}$ & $\begin{array}{l}4.00\left(\mathrm{t}, 3 \mathrm{H}, \mathrm{OCH}_{3}\right), 7.20(\mathrm{~d}, 2 \mathrm{H}, J=9.00, \mathrm{H}-3 \text { and } \mathrm{H}-5), 7.60(\mathrm{~d} 2 \mathrm{H}, \\
\mathrm{J}-9.00, \mathrm{H}-2 \text { and } \mathrm{H}-6), 8.59(\mathrm{~s}, 1 \mathrm{H}, \mathrm{CH}=\mathrm{C}), 9.00(\mathrm{~s}, 1 \mathrm{H}, \mathrm{H}-3), 9.03 \\
(\mathrm{~s}, 1 \mathrm{H}, \mathrm{H}-5),\left(\mathrm{CF}_{3} \mathrm{COOH}\right)\end{array}$ \\
\hline 12 & $\begin{array}{l}3400-2500 \text { (br., } \mathrm{NH} \text { and } \mathrm{OH}), 1725 \text { and } 1670(\mathrm{C}=\mathrm{O}) \text {, } \\
1585,1470,1445,1270,1200,810,765,740,695 .\end{array}$ & $\begin{array}{l}7.50(\mathrm{~m}, 6 \mathrm{H}, \mathrm{NH} \text { and } \mathrm{Ar}), 7.95(\mathrm{~s}, 1 \mathrm{H}, \mathrm{H}-5), 8.22(\mathrm{~s}, 1 \mathrm{H}, \mathrm{CH}=\mathrm{C}) \text {, } \\
\left(\text { DMSO-d }_{6}\right)\end{array}$ \\
\hline $13^{\mathrm{c}}$ & $\begin{array}{l}3200-2500 \text { (br., } \mathrm{OH}), 1730(\mathrm{C}=\mathrm{O}), 1595,1530\left(\mathrm{NO}_{2}\right) \\
\text { 1500, } 1400,1340\left(\mathrm{NO}_{2}\right), 1250,1225,1110,950,855, \\
815,765,750,680,670,655 .\end{array}$ & - \\
\hline 14 & $\begin{array}{l}1725 \text { and } 1710(\mathrm{C}=\mathrm{O}), 1630,1600,1545,1500,1425, \\
1400,1385,1345,1285,1230,1200,1070,1040,1030, \\
1010,955,940,870,840,760,710,690,670 .\end{array}$ & $\begin{array}{l}1.30\left(\mathrm{t}, 3 \mathrm{H}, J=7.00,-\mathrm{OCH}_{2} \mathrm{CH}_{3}\right), 1.35\left(\mathrm{t}, 3 \mathrm{H}, J=7.00,-\mathrm{OCH}_{2} \mathrm{CH}_{3}\right), \\
4.28\left(\mathrm{q}, 2 \mathrm{H}, J=7.00,-\mathrm{OCH}_{2} \mathrm{CH}_{3}\right), 4.38(\mathrm{q}, 2 \mathrm{H}, J=7.00,- \\
\left.\mathrm{OCH}_{2} \mathrm{CH}_{3}\right), 7.55(\mathrm{~m}, 6 \mathrm{H}, \mathrm{Ar} \text { and } \mathrm{H}-3), 7.86(\mathrm{~s}, 1 \mathrm{H}, \mathrm{H}-5), 8.23(\mathrm{~s}, 1 \mathrm{H}, \\
\mathrm{CH}=\mathrm{C}),\left(\mathrm{CDCl}_{3}\right)\end{array}$ \\
\hline 15 & $\begin{array}{l}1710(\mathrm{C}=\mathrm{O}), 1630,1540,1510,1450,1380,1340,1300 \\
1255,1215,1200,1060,1035,1020,1005,955,860 \\
840,795,750,695,655\end{array}$ & $\begin{array}{l}1.30\left(\mathrm{t}, 3 \mathrm{H}, J=7.00,-\mathrm{OCH}_{2} \mathrm{CH}_{3}\right), 1.35\left(\mathrm{t}, 3 \mathrm{H}, J=7.00,-\mathrm{OCH}_{2} \mathrm{CH}_{3}\right), \\
3.83\left(\mathrm{~s}, 3 \mathrm{H}, \mathrm{OCH}_{3}\right), 4.28\left(\mathrm{q}, 2 \mathrm{H}, J=7.00,-\mathrm{OCH}_{2} \mathrm{CH}_{3}\right), 4.38(\mathrm{q}, 2 \mathrm{H} \text {, } \\
\left.J=7.00,-\mathrm{OCH}_{2} \mathrm{CH}_{3}\right), 6.95\left(\mathrm{~d}, 2 \mathrm{H}, J=9.00, \mathrm{H}^{\prime}{ }^{\prime} \text { and } \mathrm{H}-5 '\right), 7.58(\mathrm{~d}, \\
\left.\left.2 \mathrm{H}, J=9.00, \mathrm{H}^{\prime}\right)^{\prime} \text { and } \mathrm{H}-6^{\prime}\right), 7.65(\mathrm{~s}, 1 \mathrm{H}, \mathrm{H}-3), 7.83(\mathrm{~s}, 1 \mathrm{H}, \mathrm{H}-5) \text {, } \\
8.13(\mathrm{~s}, 1 \mathrm{H}, \mathrm{CH}=\mathrm{C}),\left(\mathrm{CDCl}_{3}\right)\end{array}$ \\
\hline 16 & $\begin{array}{l}1730 \text { and } 1700(\mathrm{C}=\mathrm{O}), 1630,1590,1545,1515\left(\mathrm{NO}_{2}\right), \\
1500,1435,1365,1335\left(\mathrm{NO}_{2}\right), 1270,1230,1205,1010, \\
950,855,750,685,660 .\end{array}$ & $\begin{array}{l}1.32\left(\mathrm{t}, 3 \mathrm{H}, J=7.00,-\mathrm{OCH}_{2} \mathrm{CH}_{3}\right), 1.35\left(\mathrm{t}, 3 \mathrm{H}, J=7.00,-\mathrm{OCH}_{2} \mathrm{CH}_{3}\right), \\
4.30\left(\mathrm{q}, 2 \mathrm{H}, J=7.00,-\mathrm{OCH}_{2} \mathrm{CH}_{3}\right), 4.40(\mathrm{q}, 2 \mathrm{H}, J=7.00,- \\
\left.\mathrm{OCH}_{2} \mathrm{CH}_{3}\right), 7.55-8.10\left(\mathrm{~m}, 3 \mathrm{H}, \mathrm{H}-2^{\prime}, \mathrm{H}-6 \text { ' and } \mathrm{H}-3\right), 8.15-8.55(\mathrm{~m}, \\
\left.3 \mathrm{H}, \mathrm{H}-3^{\prime}, \mathrm{H}-5 \text { ' and } \mathrm{H}-5\right), 8.65(\mathrm{~s}, 1 \mathrm{H}, \mathrm{CH}=\mathrm{C}),\left(\mathrm{CF}_{3} \mathrm{COOH}\right)\end{array}$ \\
\hline 17 & $\begin{array}{l}2210(\mathrm{C} \equiv \mathrm{N}), 1610,1600,1535,1500,1450,1420,1255, \\
1245,1190,1025,955,905,860,750,685,660 .\end{array}$ & $\begin{array}{l}\text { 7.15-7.75 (m, } 11 \mathrm{H} \text {, arom. and } \mathrm{H}-3), 8.05(\mathrm{~s}, 1 \mathrm{H}, \mathrm{H}-5), 8.58(\mathrm{~s}, 1 \mathrm{H} \text {, } \\
\mathrm{CH}=\mathrm{C}),\left(\mathrm{CDCl}_{3}\right)\end{array}$ \\
\hline 18 & $\begin{array}{l}2210(\mathrm{C} \equiv \mathrm{N}), 1610,1545,1510,1300,1245,1165,1020, \\
960,825,755,685,655 .\end{array}$ & $\begin{array}{l}\left.3.84\left(\mathrm{~s}, 3 \mathrm{H}, \mathrm{OCH}_{3}\right), 7.00\left(\mathrm{~d}, 2 \mathrm{H}, J=9.00, \mathrm{H}-3^{\prime} \text { and } \mathrm{H}-5\right)^{\prime}\right), 7.20-7.80 \\
\left(\mathrm{~m}, 8 \mathrm{H}, \mathrm{Ar} \text { and } \mathrm{H}-2^{\prime} \text { and } \mathrm{H}-6^{\prime} \text { and } \mathrm{H}-3\right), 8.28(\mathrm{~s}, 1 \mathrm{H}, \mathrm{H}-5), 8.56(\mathrm{~s} \text {, } \\
1 \mathrm{H}, \mathrm{CH}=\mathrm{C}),\left(\mathrm{CDCl}_{3}-\mathrm{CF}_{3} \mathrm{COOH}\right)\end{array}$ \\
\hline 19 & $\begin{array}{l}2210(\mathrm{C} \equiv \mathrm{N}), 1610,1595,1520,1500\left(\mathrm{NO}_{2}\right), 1440,1405, \\
1385,1335\left(\mathrm{NO}_{2}\right), 1255,1190,1110,1020,950,860, \\
770,750,690 .\end{array}$ & $\begin{array}{l}\text { 7.20-7.80 (m, 9H, arom.), } 8.38(\mathrm{~s}, 1 \mathrm{H}, \mathrm{H}-3), 8.45(\mathrm{~s}, 1 \mathrm{H}, \mathrm{H}-5), 8.85 \\
(\mathrm{~s}, 1 \mathrm{H}, \mathrm{CH}=\mathrm{C}),\left(\mathrm{CF}_{3} \mathrm{COOH}\right)\end{array}$ \\
\hline 20 & $\begin{array}{l}2220(\mathrm{C} \equiv \mathrm{N}), 1710(\mathrm{C}=\mathrm{O}), 1610,1590,1535,1505, \\
1430,1400,1385,1275,1205,1010,950,870,760,725, \\
665 .\end{array}$ & $\begin{array}{l}1.40\left(\mathrm{t}, 3 \mathrm{H}, J=7.00,-\mathrm{OCH}_{2} \mathrm{CH}_{3}\right), 4.35(\mathrm{q}, 2 \mathrm{H}, J=7.00,- \\
\left.\mathrm{OCH}_{2} \mathrm{CH}_{3}\right), 7.20-7.80(\mathrm{~m}, 6 \mathrm{H}, \mathrm{Ar} \text { and } \mathrm{H}-3), 8.23(\mathrm{~s}, 1 \mathrm{H}, \mathrm{H}-5), 8.75 \\
(\mathrm{~s}, 1 \mathrm{H}, \mathrm{CH}=\mathrm{C}),\left(\mathrm{CDCl}_{3}\right)\end{array}$ \\
\hline 21 & $\begin{array}{l}2220(\mathrm{C} \equiv \mathrm{N}), 1710(\mathrm{C}=\mathrm{O}), 1610,1520,1380,1255 \\
1205,1180,1040,1010,870,830\end{array}$ & $\begin{array}{l}1.40\left(\mathrm{t}, 3 \mathrm{H}, J=7.00,-\mathrm{OCH}_{2} \mathrm{CH}_{3}\right), 3.88\left(\mathrm{~s}, 3 \mathrm{H}, \mathrm{OCH}_{3}\right), 4.35(\mathrm{q}, 2 \mathrm{H}, J \\
\left.=7.00,-\mathrm{OCH}_{2} \mathrm{CH}_{3}\right), 6.98\left(\mathrm{~d}, 2 \mathrm{H}, J=9.00, \mathrm{H}-3^{\prime} \text { and } \mathrm{H}-5\right), 7.63(\mathrm{~d}, \\
\left.2 \mathrm{H}, J=9.00, \mathrm{H}^{\prime}{ }^{\prime} \text { and } \mathrm{H}^{\prime} 6^{\prime}\right), 8.20(\mathrm{~s}, 2 \mathrm{H}, \mathrm{H}-3 \text { and } \mathrm{H}-5), 8.65(\mathrm{~s}, 1 \mathrm{H} \text {, } \\
\mathrm{CH}=\mathrm{C}),\left(\mathrm{CDCl}_{3}\right)\end{array}$ \\
\hline 22 & $\begin{array}{l}3250(\mathrm{NH}), 2220(\mathrm{C} \equiv \mathrm{N}), 1710(\mathrm{C}=\mathrm{O}), 1600,1530,1480 \\
1430,1260,1010,890,770,760,730,700,670 .\end{array}$ & $\begin{array}{l}1.35\left(\mathrm{t}, 3 \mathrm{H}, J=7.00,-\mathrm{OCH}_{2} \mathrm{CH}_{3}\right), 4.30(\mathrm{q}, 2 \mathrm{H}, J=7.00,- \\
\left.\mathrm{OCH}_{2} \mathrm{CH}_{3}\right), 7.50(\mathrm{~s}, 5 \mathrm{H}, \mathrm{Ar}), 8.17(\mathrm{~s}, 1 \mathrm{H}, \mathrm{H}-5), 8.65(\mathrm{~s}, 1 \mathrm{H}, \mathrm{CH}=\mathrm{C}) \\
\left(\mathrm{CDCl}_{3}\right)\end{array}$ \\
\hline 23 & $\begin{array}{l}2210(\mathrm{C} \equiv \mathrm{N}), 1725(\mathrm{C}=\mathrm{O}), 1590,1510\left(\mathrm{NO}_{2}\right), 1395 \\
1370,1335\left(\mathrm{NO}_{2}\right), 1305,1240,1175,1105,1025,950 \\
855,750 .\end{array}$ & $\begin{array}{l}1.30\left(\mathrm{t}, 3 \mathrm{H}, J=7.00,-\mathrm{OCH}_{2} \mathrm{CH}_{3}\right), 4.15(\mathrm{q}, 2 \mathrm{H}, J=7.00,- \\
\left.\mathrm{OCH}_{2} \mathrm{CH}_{3}\right), 7.75(\mathrm{~s}, 1 \mathrm{H}, \mathrm{H}-3), 7.80\left(\mathrm{~d}, 2 \mathrm{H}, J=9.00, \mathrm{H}-2^{\prime} \text { and } \mathrm{H}-6^{\prime}\right) \text {, } \\
7.95(\mathrm{~s}, 1 \mathrm{H}, \mathrm{H}-5), 8.25(\mathrm{~s}, 1 \mathrm{H}, \mathrm{CH}=\mathrm{C}), 8.35\left(\mathrm{~d}, 2 \mathrm{H}, J=9.00, \mathrm{H}-3^{\prime}\right. \\
\left.\text { and } \mathrm{H}^{\prime}{ }^{\prime}\right)\left(\mathrm{CDCl}_{3}\right)\end{array}$ \\
\hline 24 & $\begin{array}{l}3300-2500(\mathrm{br} ., \mathrm{OH}), 2220(\mathrm{C} \equiv \mathrm{N}), 1710(\mathrm{C}=\mathrm{O}), 1605 \text {, } \\
\text { 1590, 1540,1495, 1375, 1325, 1270,1220,1180,1020, } \\
755,705 .\end{array}$ & $\begin{array}{l}7.58(\mathrm{~m}, 5 \mathrm{H}, \mathrm{Ar}), 8.35(\mathrm{~s}, 1 \mathrm{H}, \mathrm{H}-3), 8.43(\mathrm{~s}, 1 \mathrm{H}, \mathrm{H}-5), 8.78(\mathrm{~s}, 1 \mathrm{H}, \\
\mathrm{CH}=\mathrm{C}),\left(\mathrm{CDCl}_{3}-\mathrm{CF}_{3} \mathrm{COOH}\right)\end{array}$ \\
\hline 25 & $\begin{array}{l}3200-2800 \text { (br., OH), } 2220(\mathrm{C} \equiv \mathrm{N}), 1685(\mathrm{C}=\mathrm{O}), 1600 \text {, } \\
1535,1510,1380,1290,1220,1175,755,715 .\end{array}$ & $\begin{array}{l}3.95\left(\mathrm{~s}, 3 \mathrm{H}, \mathrm{OCH}_{3}\right), 7.12(\mathrm{~d}, 2 \mathrm{H}, J=9.00, \mathrm{H}-2 \text { and } \mathrm{H}-6), 7.60(\mathrm{~d}, 2 \mathrm{H} \text {, } \\
J=9.00, \mathrm{H}-3 \text { and } \mathrm{H}-5), 8.48(\mathrm{~s}, 1 \mathrm{H}, \mathrm{H}-3), 8.56(\mathrm{~s}, 1 \mathrm{H}, \mathrm{H}-5), 8.75(\mathrm{~s}, \\
1 \mathrm{H}, \mathrm{CH}=\mathrm{C}),\left(\mathrm{CF}_{3} \mathrm{COOH}\right)\end{array}$ \\
\hline 26 & $\begin{array}{l}3290 \text { (br., } \mathrm{NH}), 3000-2400(\mathrm{br} ., \mathrm{OH}), 2220(\mathrm{C} \equiv \mathrm{N}), 1695 \\
(\mathrm{C}=\mathrm{O}), 1600,1480,1280,1265,1235,1010,950,880, \\
775,710,695,970 .\end{array}$ & $\begin{array}{l}7.71(\mathrm{~m}, 6 \mathrm{H}, \mathrm{Ar} \text { and } \mathrm{NH}), 8.49(\mathrm{~s}, 1 \mathrm{H}, \mathrm{H}-5), 9.25(\mathrm{~s}, 1 \mathrm{H}, \mathrm{CH}=\mathrm{C}) \text {, } \\
\left(\mathrm{CF}_{3} \mathrm{COOH}\right)\end{array}$ \\
\hline $27^{\mathfrak{c}}$ & $\begin{array}{l}3200(\text { br., } \mathrm{OH}), 2230(\mathrm{C} \equiv \mathrm{N}), 1710(\mathrm{C}=\mathrm{O}), 1610,1590, \\
1550,1520\left(\mathrm{NO}_{2}\right), 1495,1335\left(\mathrm{NO}_{2}\right), 1235,1080,850, \\
750,695 .\end{array}$ & - \\
\hline
\end{tabular}

${ }^{a}$ See Scheme-1; ${ }^{b}$ all strong bands; ${ }^{c}$ not very soluble in common NMR solvents.

during the reactions. As can be seen from the yields of the products (Table-1), the condensations worked well with the carbaldehydes studied. The following "active methylene" compounds were used: malonic acid, diethyl malonate, phenylacetonitrile, ethyl cyanoacetate and cyanoacetic acid. For the compounds (21-23) obtained during these reactions the yields were low but can be improved by increasing the reaction time as was the case with compounds (17-19 and 24-27).

The Knoevenagel reaction when carried out between an aldehyde and malonic acid or cyanoacetic acid in the presence of pyridine-piperidine catalyst at water bath temperature usually leads to the corresponding acrylic acid or acrylonitrile, 
respectively by undergoing decarboxylation during the reaction. The non-decarboxylated products in the earlier reported reactions have been isolated by conducting the reactions at room temperature and with ammonia as catalyst ${ }^{1}$. However, in our present work, we found that the reactions carried out with these pyrazole-4carbaldehydes with pyridine-piperidine catalyst in refluxing ethanol also gave the non-decarboxylated products (10-13 and 24-27) in good yields. No decarboxylated products were isolated from these reactions. All isolated products were well characterized through their Infrared absorption (IR) and proton magnetic resonance spectra (NMR) were consistent with the structures (Table-2).

It may be noted that some recent publications deal with the Knoevenagal reaction of pyrazole-4-carbaldehydes with malonic acid under microwave irradiation giving the corresponding acrylic acids ${ }^{26}$.

IR spectra: With the help of IR spectra (Table-2) it was easy to confirm the presence of carboxylic group in products. There were two carbonyl peaks appearing in between 1730$1675 \mathrm{~cm}^{-1}$ in case of dicarboxylated compounds and a broad peak at $3000-2500 \mathrm{~cm}^{-1}$ for -OH group. This points out that no decarboxylation has taken place during the condensation and pointing to the structures as (10-13 and 24-27). For - $\mathrm{NO}_{2}$ group containing compounds two bands appeared in the region of $1545-1495 \mathrm{~cm}^{-1}$ (asymmetric stretching) and 1350-1310 $\mathrm{cm}^{-1}$ (symmetric stretching).

${ }^{1}$ H NMR spectra: The PMR spectra were very helpful in elucidating structures of the products of condensation and readily confirmed the absence of decarboxylation during the reaction. The PMR spectra showed a singlet between 8.10 and 9.25 due to a single vinyl proton instead of two doublets expected for the two vinyl protons in case a decarboxylation would have occurred. All the products (10-13 and 24-27) displayed this feature. Other signals due to the remaining groups, aryl and pyrazole protons were also easily identified ${ }^{27}$ (Table-2).

\section{Conclusion}

Knoevenagel condensation of pyrazole-4-carbaldehydes and various "active methylene" compounds using pyridine and piperidine as base in refluxing ethanol were studied during this research work (Scheme-I). In the present study condensations with malonic acid or cyanoacetic acid did not accompany decarboxylations and the expected products retaining the carboxylic groups were isolated. IR, ${ }^{1} \mathrm{H}$ NMR and elemental analysis helped in characterizing these products. In some cases yields are improved by increasing reaction times.

\section{ACKNOWLEDGEMENTS}

The authors thank HEC for indigenous scholarships to Faryal Chaudhry, Nadia Asif, Muhammad Nasrullah and Qura Tul Ain. Cel. Jorge Ribeiro indebted to the Ministerio de Exercito, Brazil for study leave.

\section{REFERENCES}

1. (a) G. Jones, Org. React., 15, 238 (1967); (b) B. Franca and Q. Carla, Curr. Org. Synth., 9, 3139 (2012).

2. T.D. Penning, J.J. Talley, S.R. Bertenshaw, J.S. Carter, P.W. Collins, S. Docter, M.J. Graneto, L.F. Lee, J.W. Malecha, J.M. Miyashiro, R.S. Rogers, D.J. Rogier, S.S. Yu, G.D. Anderson, E.G. Burton, J.N. Cogburn, S.A. Gregory, C.M. Koboldt, W.E. Perkins, K. Seibert, A.W. Veenhuizen, Y.Y. Zhang and P.C. Isakson, J. Med. Chem., 40, 1347, (1997).

3. C.B. Vicentini, C. Romagnoli, E. Andreotti and D. Mares, J. Agric. Food Chem., 55, 10331 (2007).

4. (a) M. Biava, G.C. Porretta, A. Capelli, S. Vomero, F. Manetti, M. Botta, L. Sautebin, A.D. Rossi, F. Makovec and M. Azini, J. Med. Chem., 48, 3428 (2005); (b) M. Rovini, A. Capelli, S. Vomero, F. Manetti, M. Botta, L. Sautebin, A. D. Rossi, C. Pergola, C. Ghelardini, M. Norcini, A. Giordani, F. Makovec, P. Anzelloti and P. Patrignani, J. Med. Chem., 51, 4476 (2008); (c) M. Biava, G.C. Porretta, G. Poce, C. Battilocchio, F. Manetti, M. Botta, S. Forli, L. Sautebin, A.D. Rossi, C. Pergola, C. Ghelardini, N. Galeotti, F. Makovec, A. Giordani, P. Anzelloti, P. Patrignani and M. Anzini, J. Med. Chem., 53, 723 (2010).

5. (a) C.A. Rojahn and H.E. Kuhling, Arch. Pharm., 264, 337 (1926); (b) C.A. Rojahn and A. Seitz, Ann., 437, 297 (1924); (c) I.L. Finar and K.E. Godfrey, J. Chem. Soc., 2293 (1954).

6. W.J. Barry, J. Chem. Soc., 3851 (1961).

7. I.L. Finar and M. Manning, J. Chem. Soc., 2733 (1961).

8. A.N. Kost, L.F. Morozova and I.I. Grandberg, Zh. Org. Khim., 1, 739 (1965); Chem. Abstr., 63, 5733 (1965).

9. B.A. Porai-Koshits, I. Ya. Kvitko and E.A. Shutkova, Zh. Org. Khim., 4, 19 (1970); Chem. Abstr., 73, 3844 (1970).

10. G. Rainer and R. Riedel, Ger. Offen., 2, 141 (1972); Chem. Abstr., 77, 5453 (1972).

11. M.A. Kira, M.N. Aboul-Enein and M.I. Korkor, J. Heterocycl. Chem., 7, 25 (1970).

12. B.F. Abdel-Wahab, R.E. Khidre and A.A. Farahat, Arkivoc, 196 (2011).

13. (a) S.P. Maltseva and B.I. Stepanov, Izv. Vyssh. Ucheb. Zaved. Khim. Tekhnol., 16, 1128 (1973); Chem. Abstr., 79, 105130 (1973); (b) S.P. Maltseva, N.N. Bychkov, B.I. Stepanov, R. Ya. Mushii and V.I. Seraya, Tr. Mosk. Khim-Tekhnol. Inst., 80, 56 (1974); Chem. Abstr., 85, 62988 (1976); (c) K.R.S. Reddy, G. Srimannarayana and N.V.S. Rao, Indian Acad. Sci. A, 81, 197 (1975); Chem. Abstr., 83, 114280 (1975).

14. V.H. Nguven and B.I. Stepanov, Zh. Org. Khim., 13, 231 (1977); Chem. Abstr., 86, 139929 (1977).

15. I.M. Roushdi, A.I. El-Sebal and F.S.G. Soliman, Pharmazie, 27, 731 (1972).

16. (a) M.A.F. Elkaschef, F.M.E. Abdel-Megeid and S.M.A. Yassin, Justus, Liebigs Ann. Chem., 37 (1974); Chem. Abstr., 81, 3824 (1974); (b) A.N. Kost, L.F. Morazova and I.I. Grandberg, Zh. Org. Khim., 1, 739 (1965); Chem. Abstr., 63, 5733 (1965).

17. H. Berger, R. Gall, K. Stach, M. Thiel and W. Voemel, Ger. Offen., 2,558 (1977); Chem. Abstr., 87, 135320 (1977).

18. T.M. Efremova, K.I. Kuchkova and A.A. Semenov, Khim. Geterotsikl. Soedin., 1382 (1974); Chem. Abstr., 82, 43215 (1975).

19. G.N. Dorofeenko, A.M. Simonov and A.I. Tertova, Khim. Geterotsikl. Soedin., 568 (1973); Chem. Abstr., 79, 18629 (1973).

20. M.A. Khan and A.C.C. Freitas, Rev. Latinoamer. Quim., 13, 100 (1982); M.A. Khan and A. Mustafa, Pharmazie, 41, 813 (1986); M.A. Khan, G.P. Ellis and M.C. Pagotto, J. Heterocycl. Chem., 38, 193 (2001); S. Rashid and M.A. Khan, Pak. J. Sci. Ind. Res., 52, 289 (2009); A.Q. Ather, M.N. Tahir, M.A. Khan, K. Mehmood and F. Chaudhry, Acta Cryst. E, E66, o3170 (2010); F. Chaudhry, M.N. Tahir, M.A. Khan, A.Q. Ather and N. Asif, Acta Cryst. E., E68, o2044 (2012).

21. I.L. Finar and G.H. Lord, J. Chem. Soc., 3314 (1957).

22. J.D. Kendall and G.F. Duffin, British Patent, 797,144 (1958); Chem. Abstr., 53, 4983 (1959).

23. P. Cohen-Fernandes and C.L. Habraken, Rec. Trav. Chim., 91, 1185 (1972).

24. I.L. Finar and K.J. Saunders, J. Chem. Soc., 3862 (1965).

25. M.A. Khan and J. Ribeiro, Polish J. Chem., 61, 569 (1987).

26. V.O. Chronous B. Mykhylok and V. Mykhaylo, Synth. Commun., 34, 79 (2004); M.K. Bratenko, V.A. Chronous and M.V. Vovk, Chem. Heterocycl. Compds., 42, 1242 (2001).

27. T.J. Batterham, NMR Spectra of Simple Heterocycles, WileyIntersciences, New York, N.Y. (1973). 